4-11-2019

\title{
Estimation of Mean with two-Parameter Ratio- Product-Ratio Estimator in Double Sampling using Ancillary Information under Non-Response
}

Surya K. Pal

Vikram University, Ujjain, India, suryakantpal6676@gmail.com

Housila P. Singh

Vikram University, Ujjain, India, hpsujn@gmail.com

Follow this and additional works at: https://digitalcommons.wayne.edu/jmasm

Part of the Applied Statistics Commons, Social and Behavioral Sciences Commons, and the Statistical Theory Commons

\section{Recommended Citation}

Pal, S. K., \& Singh, H. P. (2018). Estimation of mean with two-parameter ratio-product-ratio estimator in double sampling using ancillary information under non-response. Journal of Modern Applied Statistical Methods, 17(2), eP2600. doi: 10.22237/jmasm/ 1554987299 


\section{Estimation of Mean with Two-Parameter Ratio-Product-Ratio Estimator in Double Sampling using Ancillary Information under Non-Response}

\author{
Surya K. Pal \\ Vikram University \\ Ujjain, India
}

\author{
Housila P. Singh \\ Vikram University \\ Ujjain, India
}

Ratio-product-ratio estimators with two parameters in double sampling under non-response are considered along with their properties. Practical conditions are obtained in which the suggested estimators are more proficient than other existing estimators. An example is given.

Keywords: Ancillary variable, main character, double sampling, MSE, comparison

\section{Introduction}

Non-response (NR) is an important issue that remains under constant debate amongst statistician for a variety of reasons. Some of which may be (i) refuel to answer the questionnaire, (ii) not available at home, (iii) lack of information, (iv) failure to contact, (v) unable to answer, and (vi) inaccessible. In the case of NR in double sampling, the sampling procedure due to Hansen and Hurwitz (1946) is employed for estimating the universe mean. Cochran (1977), Rao (1986), Khare and Srivastava (1993, 1995), Tabasum and Khan (2004), Singh and Kumar (2008, 2009a, 2009b, 2010a, 2010b), Singh, Kumar, and Kozak (2010), and Pal and Singh $(2016,2017)$ made their contribution towards the mean estimation of the principal variable $y$ while considering the NR at the next phase. If information (data) on the subsidiary variable $x$ is not readily available, the double sampling method is used, where a large, first-phase sample is drawn from the universe and information is collected over the variable $x$ to achieve a superior estimate of the universe mean $\bar{X}$. A second-phase sample can then be taken, and the main variable $y$ is observed. $\mathrm{Wu}$

doi: 10.22237/jmasm/1554987299 | Accepted: September 4, 2017; Published: April 11, 2019.

Correspondence: Surya K. Pal, suryakantpa16676@gmail.com 


\section{PAL \& SINGH}

and Luan (2003) discussed that the major benefit of using double sampling is the gain in high precision without significant increase in price.

\section{Methodology}

Suppose a finite universe $\mathrm{U}=\left(u_{1}, u_{2}, \ldots, u_{N}\right)$ of $N$ units. A simple random sample of size $n$ is drawn without replacement from U. Let $y_{i}$ be the value of the main variable $y$ on the unit $u_{i}(i=1,2, \ldots, N)$. In surveys on human populations, frequently $n_{1}$ units 'respond' at first attempt while the remaining $n_{2}$ units do not respond. The survey may be conducted through the mail or telephone calls, perhaps computer aided.

If NR occurs at the first attempt, Hansen and Hurwitz (1946) introduced a procedure for estimating the universe mean $\bar{Y}$ containing the subsequent steps: (i) a simple random sample of size $n$ is drawn and the questionnaire is mailed to the sampled units; (ii) a subsample of size $r=n_{2} k^{-1}(k>1)$ from the $n_{2}$ non-responding units in the initial attempt is conducted through personal interviews.

In the Hansen and Hurwitz (1946) procedure, the universe of size $N$ can be assumed to divide into two strata of size $N_{1}$ and $N_{2}=\left(N-N_{1}\right)$ of "respondents" and "non-respondents".

Let $\bar{Y}$ and $S_{y}^{2}$ be the mean and mean square of the principal character for the finite universe of $N$ units. Let $\bar{Y}_{1}$ and $S_{y_{1}}^{2}$ indicate the mean and mean square of the response group of $N_{1}$ units. Similarly, let $\bar{Y}_{2}$ and $S_{y_{2}}^{2}$ indicate the mean and mean square/variance of the NR group $N_{2}$.

The universe mean $\bar{Y}$ of the principal variable $y$ is given as

$$
\bar{Y}=D_{1} \bar{Y}_{1}+D_{2} \bar{Y}_{2}
$$

with $D_{1}=\left(N_{1} / N\right)$ and $D_{2}=\left(N_{2} / N\right)$. For $\bar{Y}$, the unbiased estimator is

$$
\bar{y}^{*}=d_{1} \bar{y}_{1}+d_{2} \bar{y}_{2 r}
$$

with $d_{1}=\left(n_{1} / n\right), d_{2}=\left(n_{2} / n\right)$, and $\bar{y}_{1}$ and $\bar{y}_{2}$ are the sample means depend upon $n_{1}$ and $r$ units. The variance of $\bar{y}^{*}$ is

$$
\mathrm{V}\left(\bar{y}^{*}\right)=\bar{Y}^{2}\left[\lambda C_{y}^{2}+\lambda^{*} C_{y(2)}^{2}\right]
$$


(Cochran, 1977, p. 371), where $\lambda=(1-f) n^{-1}=\left(n^{-1}-N^{-1}\right), f$ is the sampling fraction, and $\lambda^{*}=n^{-1} D_{2}(k-1)$. We also define $C_{x}=S_{x} / \bar{X}$ and $C_{x(2)}=S_{x(2)} / \bar{X}$ as the coefficients of variation of the whole universe and NR group, respectively.

\section{The Double Sampling Method and Estimators}

If the list of units is available but $\bar{X}$ is not known, insert $\bar{x}^{\prime}$ based on a large sample of size $n^{\prime}$ in place of $\bar{X}$. The sampling design will be as follows: (1) choose a large sample of size $n^{\prime}$ in the first-phase via a simple random sampling without replacement (SRSWOR) method and observed $x$ variable. (2) From the selected $n^{\prime}$ first-phase units, we select a second-phase sample of $n$ via SRSWOR and observe that $n_{1}$ and $n_{2}$ observations are responding and not-responding, respectively. Collect information on $y$ for $n_{1}$ responding units. (3) From the $n_{2}$ NR observations, select a sub-sample of size $r=n_{2} k^{-1}(k>1)$ using SRSWOR by making an extra effort and observe the character $y$ for these $r$ chosen units. There are $n^{\prime}$ observations on the $x$ variable. Of the $n$ second-phase units there are $n_{1}$ observations on the $y$ variable from units who respond, and also $r$ observations on the sub-sample selected from the $n_{2}$ NR units of the second-phase sample. Let $\bar{x}^{\prime}$ be the sample mean of $x$ based on a preliminary large sample $n^{\prime}$. Using the information on $x$ when $\bar{X}$ is not known, consider two classes of estimators for $\bar{Y}$ in two unusual situations, which are as follows:

Situation I: The case when $\bar{X}$ is unknown and incomplete information is available on the main variable $y$ and the supplementary variable $x$. In this situation, we use $\left(n_{1}+r\right)$ responding units for $y$ and $x$ from the sample of size $n$ and $\bar{x}^{\prime}$ to estimate $\bar{X}$. Khare and Srivastava $(1993,1995)$ and Tabasum and Khan (2004) suggested the following two-phase sampling ratio and product type estimators for $\bar{Y}$ :

$$
\begin{gathered}
T_{\mathrm{R} 1 d}=z^{-1} \bar{y}^{*}, \\
T_{\mathrm{Pl} d}=z \bar{y}^{*},
\end{gathered}
$$

where $z=\left(\bar{x}^{*} / \bar{x}^{\prime}\right)$.

Up to order $n^{-1}$, the expression for bias and mean squared error (MSE) of $T_{\mathrm{R} 1 d}$ and of $T_{\mathrm{P} 1 d}$ are as follows: 


\section{PAL \& SINGH}

$$
\begin{gathered}
\mathrm{B}\left(T_{\mathrm{R} 1 d}\right)=\bar{Y}\left\{\theta C_{x}^{2}+\lambda^{*} C_{x(2)}^{2}\right\}\left(1-R_{d}^{*}\right), \\
\operatorname{MSE}\left(T_{\mathrm{R} 1 d}\right)=\bar{Y}^{2}\left[\left(\lambda C_{y}^{2}+\lambda^{*} C_{y(2)}^{2}\right)+\left\{\theta C_{x}^{2}+\lambda^{*} C_{x(2)}^{2}\right\}\left(1-2 R_{d}^{*}\right)\right], \\
\mathrm{B}\left(T_{\mathrm{P} 1 d}\right)=\bar{Y}\left[\theta C_{x}^{2}+\lambda^{*} C_{x(2)}^{2}\right] R_{d}^{*} \\
\operatorname{MSE}\left(T_{\mathrm{P} 1 d}\right)=\bar{Y}^{2}\left[\left(\lambda C_{y}^{2}+\lambda^{*} C_{y(2)}^{2}\right)+\left\{\theta C_{x}^{2}+\lambda^{*} C_{x(2)}^{2}\right\}\left(1+2 R_{d}^{*}\right)\right],
\end{gathered}
$$

where

$$
R_{d}^{*}=\left[\theta C C_{x}^{2}+\lambda^{*} C_{(2)} C_{x(2)}^{2}\right] /\left[\theta C_{x}^{2}+\lambda^{*} C_{x(2)}^{2}\right]
$$

and $\theta=\left(n^{-1}-n^{\prime-1}\right)$ for

$$
C=\rho_{y x}\left(\frac{C_{y}}{C_{x}}\right), C_{(2)}=\rho_{y x(2)}\left(\frac{C_{y(2)}}{C_{x(2)}}\right), \rho_{y x}=\left(\frac{S_{y x}}{S_{y} S_{x}}\right), \rho_{y x(2)}=\left(\frac{S_{y x(2)}}{S_{y(2)} S_{x(2)}}\right),
$$

where $S_{y x}$ and $S_{y x(2)}$ are the covariance of the entire group and NR group, respectively.

From (3) and (7),

$$
\mathrm{V}\left(\bar{y}^{*}\right)-\operatorname{MSE}\left(T_{\mathrm{R} 1 d}\right)=\bar{Y}^{2}\left\{\theta C_{x}^{2}+\lambda^{*} C_{x(2)}^{2}\right\}\left(2 R_{d}^{*}-1\right)
$$

It follows from (10) that the estimator $T_{\mathrm{R} 1 d}$ is more accurate than $\bar{y}^{*}$ if

$$
R_{d}^{*}>(1 / 2)
$$

In a similar fashion it can be shown that the estimator $T_{\mathrm{P} 1 d}$ is more accurate than $\bar{y}^{*}$ if

$$
R_{d}^{*}<-(1 / 2) \text {. }
$$




\section{ESTIMATION OF MEAN WITH TWO-PARAMETER...}

Observing conditions (11) and (12), the conventional unbiased estimator $\bar{y}^{*}$ is to be preferred over the ratio estimator $T_{\mathrm{R} 1 d}$ and product estimator $T_{\mathrm{P} 1 d}$ if

$$
-(1 / 2) \leq R_{d}^{*} \leq(1 / 2)
$$

Situation II: The case when $\bar{X}$ is unknown and incomplete information on $y$ and complete information on $x$ is available. In this situation, using information on the responding units $\left(n_{1}+r\right)$ on $y$ and complete information on $x$ from $n$, the two-phase sampling estimators for $\bar{Y}$ are

$$
\begin{gathered}
T_{\mathrm{R} 2 d}=v^{-1} \bar{y}^{*}, \\
T_{\mathrm{P} 2 d}=v \bar{y}^{*},
\end{gathered}
$$

where $v=\left(\bar{x} / \bar{x}^{\prime}\right)$.

Up to order $n^{-1}$, the bias and MSE of $T_{\mathrm{R} 2 d}$ and $T_{\mathrm{P} 2 d}$ are as follows:

$$
\begin{gathered}
\mathrm{B}\left(T_{\mathrm{R} 2 d}\right)=\bar{Y} \theta C_{x}^{2}(1-C), \\
\operatorname{MSE}\left(T_{\mathrm{R} 2 d}\right)=\bar{Y}^{2}\left[\left(\lambda C_{y}^{2}+\lambda^{*} C_{y(2)}^{2}\right)+\theta C_{x}^{2}(1-2 C)\right], \\
\mathrm{B}\left(T_{\mathrm{P} 2 d}\right)=\bar{Y} \theta C C_{x}^{2}, \\
\operatorname{MSE}\left(T_{\mathrm{R} 2 d}\right)=\bar{Y}^{2}\left[\left(\lambda C_{y}^{2}+\lambda^{*} C_{y(2)}^{2}\right)+\theta C_{x}^{2}(1+2 C)\right] .
\end{gathered}
$$

The estimators $T_{\mathrm{R} 2 d}$ and $T_{\mathrm{P} 2 d}$ are respectively better than $\bar{y}^{*}$ if

$$
C>(1 / 2)
$$

and

$$
C<-(1 / 2)
$$

However, $\bar{y}^{*}$ is to be preferred over $T_{\mathrm{R} 1 d}$ and $T_{\mathrm{P} 1 d}$ if 


\section{PAL \& SINGH}

$$
-(1 / 2) \leq C \leq(1 / 2)
$$

Taking motivation from Chami, Singh, and Thomas (2012), consider a twoparameter ratio-product-ratio (RPR) estimator and its properties in double sampling with non-respondents in two different situations.

\section{The Suggested Two-Parameter RPR Estimator}

Consider a two-parameter RPR estimator in two-phase sampling in two situations (i.e. Case I and Case II).

\section{Case I: There Is Non-Response on $y$ as Well as on $x$}

In this situation, for estimating the $\bar{Y}$ of $y$, we propose the following two-parameter RPR estimator:

$$
T_{d(\alpha, \beta)}=\left[\alpha\left\{\frac{(1-\beta) z+\beta}{\beta z+(1-\beta)}\right\}+(1-\alpha)\left\{\frac{\beta z+(1-\beta)}{(1-\beta) z+\beta}\right\}\right] \bar{y}^{*}
$$

where $\alpha, \beta$ are real constants (see Chami et al., 2012). The goal is to derive values for these constants $\alpha, \beta$ such that the bias and/or the MSE of $T_{d(\alpha, \beta)}$ are minimal. The two parameters $\alpha$ and $\beta$ may be used to obtain an asymptotically optimum estimator (AOE) $T_{d(\alpha, \beta)}^{(0)}$ that is (up to order $n^{-1}$ ) both unbiased and has minimal MSE. The

estimator $T_{d(\alpha, \beta)}^{(0)}$ corrects the limitations of the commonly used estimators $\bar{y}^{*}, T_{\mathrm{R} 1 d}$, and $T_{\mathrm{P} 1 d}$, which are to be used for a specific range of the parameters $\left(C, C_{(2)}\right.$, or $\left.R_{d}^{*}\right)$ and, in addition, out-performs the traditional estimators by having the minimum MSE.

$T_{d(\alpha, \beta)}=T_{d(1-\alpha, 1-\beta)}$, meaning the estimator $T_{d(\alpha, \beta)}$ is invariant under a point reflection through the point $(\alpha, \beta)=(1 / 2,1 / 2)$. In the point of symmetry $(\alpha, \beta)=(1 / 2,1 / 2)$, the estimator reduces to $\bar{y}^{*}$ due to Hansen and Hurwitz (1946). In fact, on the entire line $\beta=1 / 2$, the suggested estimator reduces to $\bar{y}^{*}$. For $(\alpha, \beta)=(1,0)$ or $(\alpha, \beta)=(0,1)$, the recommended estimator $T_{d(\alpha, \beta)}$ reduces to $T_{d(1,0)}=T_{d(0,1)}=\left(\bar{x}^{*} \bar{y}^{*}\right) / \bar{x}^{\prime}=T_{\mathrm{P} 1 d}$, while for $(\alpha, \beta)=(0,0)$ or $(\alpha, \beta)=(1,1)$, it reduces to the ratio estimator $T_{d(0,0)}=T_{d(1,1)}=\left(\bar{y}^{*} \bar{x}^{\prime}\right) / \bar{x}^{*}=T_{\mathrm{R} 1 d}$.

All the three estimators $\bar{y}^{*}, T_{\mathrm{R} 1 d}$, and $T_{\mathrm{P} 1 d}$ can be obtained from the proposed estimate $T_{d(\alpha, \beta)}$ by using suitable values of the parameters $(\alpha, \beta)$. Consider estimator 


\section{ESTIMATION OF MEAN WITH TWO-PARAMETER...}

(23) and compare it to the three estimators $\bar{y}^{*}, T_{\mathrm{R} 1 d}$, and $T_{\mathrm{P} 1 d}$ as follows: In order to derive the bias of $T_{d(\alpha, \beta)}$ up to $\mathrm{O}\left(n^{-1}\right)$, write

$$
e_{0}=\frac{\bar{y}^{*}-\bar{Y}}{\bar{Y}}, \quad e_{1}=\frac{\bar{x}^{*}-\bar{X}}{\bar{X}}, \quad \text { and } \quad e_{1}^{\prime}=\frac{\bar{x}^{\prime}-\bar{X}}{\bar{X}}
$$

such that $\mathrm{E}\left(e_{i}\right)=0$ for $i=0,1$ and $\mathrm{E}\left(e_{1}^{\prime}\right)=0$, with relative variances

$$
\mathrm{E}\left(e_{0}^{2}\right)=\left[\lambda C_{y}^{2}+\lambda^{*} C_{y(2)}^{2}\right], \quad \mathrm{E}\left(e_{1}^{2}\right)=\left[\lambda C_{x}^{2}+\lambda^{*} C_{x(2)}^{2}\right], \quad \mathrm{E}\left(e_{1}^{\prime 2}\right)=\lambda^{\prime} C_{x}^{2},
$$

where $\lambda^{\prime}=\left(n^{\prime-1}-N^{-1}\right)$.

Also,

$$
\mathrm{E}\left(e_{0} e_{1}\right)=\left[\lambda \rho_{y x} C_{y} C_{x}+\lambda^{*} \rho_{y x(2)} C_{y(2)} C_{x(2)}\right], \mathrm{E}\left(e_{0} e_{1}^{\prime}\right)=\lambda^{\prime} \rho_{y x} C_{y} C_{x}, \mathrm{E}\left(e_{1} e_{1}^{\prime}\right)=\lambda^{\prime} C_{x}^{2} .
$$

Express (23) as

$$
T_{d(\alpha, \beta)}=\bar{Y}\left(1+e_{0}\right)\left[\alpha \frac{\left(1+(1-\beta) e_{1}+\beta e_{1}^{\prime}\right)}{\left(1+\beta e_{1}+(1-\beta) e_{1}^{\prime}\right)}+(1-\alpha) \frac{\left(1+\beta e_{1}+(1-\beta) e_{1}^{\prime}\right)}{\left(1+(1-\beta) e_{1}+\beta e_{1}^{\prime}\right)}\right]
$$

From (24),

$$
\begin{gathered}
T_{d(\alpha, \beta)} \cong \bar{Y}\left[1+e_{0}-(1-2 \alpha)(1-2 \beta)\left(e_{0}-e_{1}^{\prime}+e_{0} e_{1}-e_{0} e_{1}^{\prime}\right)+(1-\alpha-\beta)(1-2 \beta) e_{1}^{2}\right. \\
\left.+(\alpha-\beta)(1-2 \beta) e_{1}^{\prime 2}-\left(1-4 \beta+4 \beta^{2}\right) e_{1} e_{1}^{\prime}\right]
\end{gathered}
$$

or

$$
\begin{aligned}
& \left(T_{d(\alpha, \beta)}-\bar{Y}\right) \cong \bar{Y}\left[e_{0}-(1-2 \alpha)(1-2 \beta)\left(e_{0}-e_{1}^{\prime}+e_{0} e_{1}-e_{0} e_{1}^{\prime}\right)\right. \\
& \left.\quad+(1-\alpha-\beta)(1-2 \beta) e_{1}^{2}+(\alpha-\beta)(1-2 \beta) e_{1}^{\prime 2}-\left(1-4 \beta+4 \beta^{2}\right) e_{1} e_{1}^{\prime}\right]
\end{aligned}
$$

Taking expectations together with (25), the expected bias of $T_{d(\alpha, \beta)}$ is obtained as 


\section{PAL \& SINGH}

$$
\begin{aligned}
\mathrm{B}\left(T_{d(\alpha, \beta)}\right) & =\mathrm{E}\left(T_{d(\alpha, \beta)}-\bar{Y}\right) \\
& \cong \bar{Y}(1-2 \beta)\left\{\theta C_{x}^{2}+\lambda^{*} C_{x(2)}^{2}\right\}\left[(1-\alpha-\beta)-(1-2 \alpha) R_{d}^{*}\right]
\end{aligned}
$$

Equating (26) to zero,

$$
\beta=1 / 2 \quad \text { or } \quad \beta=\left[1-\alpha-(1-2 \alpha) R_{d}^{*}\right]
$$

The proposed RPR estimator $T_{d(\alpha, \beta)}$, substituted with the value of $\beta$ from (27), becomes an approximately unbiased estimator for $\bar{Y}$. Furthermore, as the sample size $n$ is very large, the bias of $T_{d(\alpha, \beta)}$ will be negligible. If there is response not present on $x$ the result in (27) reduces to

$$
\beta=1 / 2 \quad \text { or } \quad \beta=[1-\alpha-(1-2 \alpha) C] \text {. }
$$

Squaring (25) obtains the approximate expression

$$
\begin{gathered}
\left(T_{d(\alpha, \beta)}-\bar{Y}\right)^{2} \cong \bar{Y}^{2}\left[e_{0}^{2}+(1-2 \alpha)^{2}(1-2 \beta)^{2}\left(e_{1}^{2}-e_{1}^{\prime 2}-e_{1} e_{1}^{\prime}\right)\right. \\
\left.-2(1-2 \alpha)(1-2 \beta)\left(e_{0} e_{1}-e_{0} e_{1}^{\prime}\right)\right]
\end{gathered}
$$

The approximate MSE of $T_{d(\alpha, \beta)}$ is obtained as

$$
\begin{aligned}
\operatorname{MSE}\left(T_{d(\alpha, \beta)}\right)=\bar{Y}^{2}\left[\left(\lambda C_{y}^{2}+\lambda^{*} C_{y(2)}^{2}\right)+(1-2 \alpha)^{2}(1-2 \beta)^{2}\left\{\theta C_{x}^{2}+\lambda^{*} C_{x(2)}^{2}\right\}\right. & \left.-2(1-2 \alpha)(1-2 \beta)\left\{\theta \rho_{y x} C_{y} C_{x}+\lambda^{*} \rho_{y x(2)} C_{y(2)} C_{x(2)}\right\}\right] \\
= & \bar{Y}^{2}\left[\left(\lambda C_{y}^{2}+\lambda^{*} C_{y(2)}^{2}\right)\right. \\
+ & \left.(1-2 \alpha)(1-2 \beta)\left\{\theta C_{x}^{2}+\lambda^{*} C_{x(2)}^{2}\right\}\left\{(1-2 \alpha)(1-2 \beta)-2 R_{d}^{*}\right\}\right]
\end{aligned}
$$

Taking the gradient $\nabla=(\partial / \partial \alpha, \partial / \partial \beta)$ of $(30)$,

$$
\begin{aligned}
\nabla \operatorname{MSE}\left(T_{d(\alpha, \beta)}\right)= & 4 \bar{Y}^{2}\left\{\theta C_{x}^{2}+\lambda^{*} C_{x(2)}^{2}\right\}\left[(1-2 \alpha)(1-2 \beta)-R_{d}^{*}\right] \\
& (1-2 \alpha, 1-2 \beta)
\end{aligned}
$$




\section{ESTIMATION OF MEAN WITH TWO-PARAMETER...}

Equating (31) to zero to obtain the critical points, we obtain the following solutions:

$$
\alpha=1 / 2, \beta=1 / 2
$$

or

$$
(1-2 \alpha)(1-2 \beta)=R_{d}^{*}
$$

The critical point in (32) is a saddle point unless $R_{d}^{*}=0$, in which case a local minimum is obtained. However, the critical points obtained in (33) give the equation of the hyperbola symmetric through $(\alpha, \beta)=(1 / 2,1 / 2)$. The minimum mean squared error (MMSE) of $T_{d(\alpha, \beta)}$ is obtained as

$$
\operatorname{MMSE}\left(T_{d(\alpha, \beta)}\right)=\bar{Y}^{2}\left[\left(\lambda C_{y}^{2}+\lambda^{*} C_{y(2)}^{2}\right)-\left\{\theta C_{x}^{2}+\lambda^{*} C_{x(2)}^{2}\right\} R_{d}^{* 2}\right]
$$

which is independent of $\alpha$ and $\beta$.

Theorem 1. Up to $\mathrm{O}\left(n^{-1}\right)$,

$$
\operatorname{MSE}\left(T_{d(\alpha, \beta)}\right) \geq \bar{Y}^{2}\left[\left(\lambda C_{y}^{2}+\lambda^{*} C_{y(2)}^{2}\right)-\left\{\theta C_{x}^{2}+\lambda^{*} C_{x(2)}^{2}\right\} R_{d}^{* 2}\right]
$$

if $(1-2 \alpha)(1-2 \beta)=R_{d}^{*}$.

This is the minimal possible MSE up to order $n^{-1}$ for a wide family of estimators to which the estimator (23) belongs, for instance, for estimators

$$
t_{d h}=\bar{y}^{*} \mathrm{~h}(z)
$$

$\mathrm{h}($.) being a function of $z$ such that $\mathrm{h}(1)=1$ and also satisfies certain regularity conditions similar to those given in Srivastava (1971). Whatever value of $R_{d}^{*}$ in (30) has, we are always able to select an $\mathrm{AOE} T_{d(\alpha, \beta)}^{(0)}$ from the two-parameter family in (23).

Further, putting (32) into $T_{d(\alpha, \beta)}^{(0)}$ yields $\bar{y}^{*}$ of $\bar{Y}$. Thus, the MSE of $\bar{y}^{*}$ is 


\section{PAL \& SINGH}

$$
\operatorname{MSE}\left(T_{d(1 / 2,1 / 2)}\right)=\operatorname{MSE}\left(\bar{y}^{*}\right)=\bar{Y}^{2}\left[\lambda C_{y}^{2}+\lambda^{*} C_{y(2)}^{2}\right]
$$

Remark 1. When NR occurs on both $y$ and $x$ with unknown $\bar{X}$, an alternative to a two-parameter RPR estimator for $\bar{Y}$ is defined by

$$
T_{d(\alpha, \beta)}^{(1)}=\left[\alpha z^{(1-2 \beta)}+(1-\alpha) z^{(2 \beta-1)}\right] \bar{y}^{*}
$$

The estimator $T_{d(\alpha, \beta)}^{(1)}$ has the same bias and MSE up to order $n^{-1}$ as $T_{d(\alpha, \beta)}^{(0)}$ in (23).

The class of estimators $T_{d(\alpha, \beta)}^{(1)}$ is further generalized along the lines of Singh, Solanki, and Singh (2016) as

$$
T_{d(\alpha, \beta)}^{(2)}=\left[\alpha z^{*(1-2 \beta)}+(1-\alpha) z^{*(2 \beta-1)}\right] \bar{y}^{*},
$$

where $z^{*}=\left(a \bar{x}^{*}+b\right) /\left(a \bar{x}^{\prime}+b\right),(\alpha, \beta)$ are the same defined in Chami et al. (2012, p. $2)$, and $a(\neq 0)$ and $b$ are either real or the functions of the known parameters associated with $x$ and $y$ or both $(x, y)$.

\section{Efficiency Comparison and Choice of Parameters}

Comparing the MSE of $\bar{y}^{*}$ to $T_{d(\alpha, \beta)}$, observe the following from (3) or (36) and from (30):

$$
\begin{array}{r}
\mathrm{V}\left(\bar{y}^{*}\right)-\operatorname{MSE}\left(T_{d(\alpha, \beta)}\right)=-\bar{Y}^{2}\left[(1-2 \alpha)(1-2 \beta)\left(\theta C_{x}^{2}+\lambda^{*} C_{x(2)}^{2}\right)\right. \\
\left.+\{(1-2 \alpha)(1-2 \beta)\}-2 R_{d}^{*}\right]
\end{array}
$$

which is positive if

$$
(1-2 \alpha)(1-2 \beta)\left[2 R_{d}^{*}-(1-2 \alpha)(1-2 \beta)\right]>0 .
$$

Therefore

(i) $\quad \alpha>\frac{1}{2}, \beta>\frac{1}{2}$ and $R_{d}^{*}>\frac{1}{2}(1-2 \alpha)(1-2 \beta)$, 


\section{ESTIMATION OF MEAN WITH TWO-PARAMETER...}

(ii) $\quad \alpha<\frac{1}{2}, \beta>\frac{1}{2}$ and $R_{d}^{*}<\frac{1}{2}(1-2 \alpha)(1-2 \beta)$,

(iii) $\quad \alpha>\frac{1}{2}, \beta<\frac{1}{2}$ and $R_{d}^{*}<\frac{1}{2}(1-2 \alpha)(1-2 \beta)$, or

(iv) $\alpha<\frac{1}{2}, \beta<\frac{1}{2}$ and $R_{d}^{*}>\frac{1}{2}(1-2 \alpha)(1-2 \beta)$.

The conventional unbiased estimator $y^{*}$ is to be preferred if $-1 / 2 \leq R_{d}^{*} \leq 1 / 2$. Combining (i) to (iv) with $-1 / 2 \leq R_{d}^{*} \leq 1 / 2$, obtain the following explicit range:

(v) If $0<R_{d}^{*} \leq \frac{1}{2}$ and $\beta>\frac{1}{2}$, then $\frac{1}{2}<\alpha<\frac{\left(2 \beta+2 R_{d}^{*}-1\right)}{2(2 \beta-1)}$ from (i).

(vi) If $-\frac{1}{2} \leq R_{d}^{*}<0$ and $\beta>\frac{1}{2}$, then $\frac{\left(2 \beta+2 R_{d}^{*}-1\right)}{2(2 \beta-1)}<\alpha<\frac{1}{2}$ from (ii).

(vii) If $-\frac{1}{2} \leq R_{d}^{*}<0$ and $\beta<\frac{1}{2}$, then $\frac{1}{2}<\alpha<\frac{\left(2 \beta+2 R_{d}^{*}-1\right)}{2(2 \beta-1)}$ from (iii).

(viii) If $0<R_{d}^{*} \leq \frac{1}{2}$ and $\beta<\frac{1}{2}$, then $\frac{\left(2 \beta+2 R_{d}^{*}-1\right)}{2(2 \beta-1)}<\alpha<\frac{1}{2}$ from (iv).

Comparing the MSE of $T_{\mathrm{R} 1 d}$ and $T_{d(\alpha, \beta)}$, from (7) and (36),

$$
\begin{aligned}
\operatorname{MSE}( & \left.T_{\mathrm{R} 1 d}\right)-\operatorname{MSE}\left(T_{d(\alpha, \beta)}\right) \\
& =4 \bar{Y}^{2}\left\{\theta C_{x}^{2}+\lambda^{*} C_{x(2)}^{2}\right\}\left[(2 \alpha \beta-\alpha-\beta)\left\{R_{d}^{*}-1-(2 \alpha \beta-\alpha-\beta)\right\}\right]
\end{aligned}
$$

which is positive if

$$
(2 \alpha \beta-\alpha-\beta)\left\{R_{d}^{*}-1-(2 \alpha \beta-\alpha-\beta)\right\}>0 .
$$

Therefore,

$$
R_{d}^{*}-1>(2 \alpha \beta-\alpha-\beta)>0
$$




\section{PAL \& SINGH}

or

$$
R_{d}^{*}-1<(2 \alpha \beta-\alpha-\beta)<0
$$

Hence, from (42), when $R_{d}^{*}>1$,

$$
\begin{aligned}
& \text { If } \beta<\frac{1}{2} \text {, then } \frac{\left(\beta+R_{d}^{*}-1\right)}{(2 \beta-1)}<\alpha<\frac{\beta}{(2 \beta-1)} \text {. } \\
& \text { If } \beta>\frac{1}{2} \text {, then } \frac{\beta}{(2 \beta-1)}<\alpha<\frac{\left(\beta+R_{d}^{*}-1\right)}{(2 \beta-1)} \text {. }
\end{aligned}
$$

Further, from (42), when $1 / 2<R_{d}^{*}<1$ we have

$$
\begin{aligned}
& \text { If } \beta<\frac{1}{2} \text {, then } \frac{\beta}{(2 \beta-1)}<\alpha<\frac{\left(\beta+R_{d}^{*}-1\right)}{(2 \beta-1)} \text {. } \\
& \text { If } \beta>\frac{1}{2} \text {, then } \frac{\left(\beta+R_{d}^{*}-1\right)}{(2 \beta-1)}<\alpha<\frac{\beta}{(2 \beta-1)} \text {. }
\end{aligned}
$$

Comparing the MSE of $T_{\mathrm{P} 1 d}$ to $T_{d(\alpha, \beta)}$, from (9) and (36),

$$
\begin{aligned}
\operatorname{MSE}( & \left.T_{\mathrm{P} 1 d}\right)-\operatorname{MSE}\left(T_{d(\alpha, \beta)}\right) \\
& =4 \bar{Y}^{2}\left\{\theta C_{x}^{2}+\lambda^{*} C_{x(2)}^{2}\right\}\left[(1+2 \alpha \beta-\alpha-\beta)\left\{R_{d}^{*}-(2 \alpha \beta-\alpha-\beta)\right\}\right]
\end{aligned}
$$

The expression (47) is positive if

$$
(1+2 \alpha \beta-\alpha-\beta)\left[R_{d}^{*}-(2 \alpha \beta-\alpha-\beta)\right]>0 .
$$

Obtain the following two cases:

$$
R_{d}^{*}>(2 \alpha \beta-\alpha-\beta)>-1 \text { if both factors in (47) are positive or }
$$




\section{ESTIMATION OF MEAN WITH TWO-PARAMETER...}

$$
-1, R_{d}^{*}<(2 \alpha \beta-\alpha-\beta) \text { if both factors in (47) are negative. }
$$

\section{Unbiased Asymptotically Optimum Estimators}

Solving the two equations (27) and (33), calculate the parameters $\alpha$ and $\beta$, where the proposed class of estimators $T_{d(\alpha, \beta)}$ turns out to be, at least up to first degree of approximation, an unbiased AOE. Obtain a line

$$
\beta=\frac{1}{2}, \quad\left(C, C_{(2)}\right)=(0,0) \text { or } R_{d}^{*}=0
$$

(recall that on this line the recommended family $T_{d(\alpha, \beta)}$ always reduces to $\bar{y}^{*}$ ) and a curve $\left(\alpha^{*}\left(R_{d}^{*}\right), \beta^{*}\left(R_{d}^{*}\right), R_{d}^{*}\right) \in \mathrm{R}^{3}$ in the parameter space with

$$
\alpha^{*}\left(R_{d}^{*}\right)=\frac{1}{2}\left(1 \pm \sqrt{\frac{R_{d}^{*}}{2 R_{d}^{*}-1}}\right), \quad \beta^{*}\left(R_{d}^{*}\right)=\frac{1}{2}\left(1 \pm \sqrt{R_{d}^{*}\left(2 R_{d}^{*}-1\right)}\right)
$$

Inserting the values of $\alpha^{*}\left(R_{d}^{*}\right)$ and $\beta^{*}\left(R_{d}^{*}\right)$ given by (52) in (23), obtain the estimator of $\bar{Y}$ as

$$
\begin{aligned}
T_{d\left(R^{*}\right)} & =T_{d\left(\alpha^{*}\left(R_{d}^{*}\right), \beta^{*}\left(R_{d}^{*}\right)\right)} \\
& =\bar{y}^{*}\left[\frac{2\left(R_{d}^{*}+1\right) \bar{x}^{\prime 2}-2\left(R_{d}^{*}-1\right) \bar{x}^{* 2}+\left(2 R_{d}^{* 2}-R_{d}^{*}-1\right)\left(\bar{x}^{\prime}-\bar{x}^{*}\right)^{2}}{4 \bar{x}^{\prime} \bar{x}^{*}-\left(2 R_{d}^{* 2}-R_{d}^{*}-1\right)\left(\bar{x}^{\prime}-\bar{x}^{*}\right)^{2}}\right]
\end{aligned}
$$

The denominator vanishes if

$$
R_{d}^{*}=0.25\left[1 \pm \sqrt{9+\frac{32 \bar{x}^{\prime} \bar{x}^{*}}{u^{2}}}\right], \quad u=\left(\bar{x}^{*}-\bar{x}^{\prime}\right)
$$

Thus,

$$
\mathrm{B}\left(T_{d\left(R^{*}\right)}\right)=0
$$

and 


$$
\begin{aligned}
\operatorname{MSE}\left(T_{d\left(R^{*}\right)}\right) & =\bar{Y}^{2}\left[\left(\lambda C_{y}^{2}+\lambda^{*} C_{y(2)}^{2}\right)-\frac{\left\{\theta C C_{x}^{2}+\lambda^{*} C_{(2)} C_{x(2)}^{2}\right\}^{2}}{\theta C_{x}^{2}+\lambda^{*} C_{x(2)}^{2}}\right] \\
& =\mathrm{V}\left(\bar{y}^{*}\right)\left(1-\rho^{\prime * 2}\right)
\end{aligned}
$$

where $\rho^{\prime *}$ is the correlation coefficient between $\bar{y}^{*}$ and $u$.

The estimator $T_{d\left(R^{*}\right)}$ in (53) is an unbiased AOE. One might be interested to know whether inside $0<R_{d}^{*} \leq 1 / 2$ there is a choice of real parameters $(\alpha, \beta) \in \mathrm{R}^{2}$ such that an AOE with small bias is obtained. Putting (33) in (27) yields the firstdegree approximation of the bias of an AOE:

$$
\mathrm{B}\left(T_{d(\alpha, \beta)}^{*}\right)=\bar{Y}^{2}\left[\theta C_{x}^{2}+\lambda^{*} C_{x(2)}^{2}\right]\left[R_{d}^{*}\left(1-2 R_{d}^{*}\right)+(1-2 \beta)^{2}\right] .
$$

It follows from (33) and (55) the bias can only be made zero if $R_{d}^{*} \leq 0$ or $R_{d}^{*} \geq 1 / 2$. Otherwise, there is always a positive contribution coming from the term $R_{d}^{*}\left(1-2 R_{d}^{*}\right)$ that does not vanish regardless of what is chosen for (see Chami et al., 2012, p. 10).

\section{Case II: There is Non-Response on $y$ Only, Complete Information is Available for a Sample of Size $n$ on the Subsidiary Variable $x$}

If NR occurs only on $y$ and information lacks about $\bar{X}$, a two-parameter RPR estimator suggested for $\bar{Y}$ is

$$
P_{d(\eta, \delta)}=\left[\eta\left\{\frac{(1-\delta) v+\delta}{\delta v+(1-\delta)}\right\}+(1-\eta)\left\{\frac{\bar{x} v+(1-\delta)}{(1-\delta) v+\delta}\right\}\right] \bar{y}^{*}
$$

where $(\eta, \delta)$ are real constants.

The objective is to obtain values for these scalars $(\eta, \delta)$ such that bias or the MSE of $P_{d(\eta, \delta)}$ are minimal. Note that $P_{d(\eta, \delta)}=P_{d(1-\eta, 1-\delta)}$; that is, the estimator $P_{d(\eta, \delta)}$ is invariant under a point reflection through the point $(\eta, \delta)=(1 / 2,1 / 2)$. In the point of symmetry $(\eta, \delta)=(1 / 2,1 / 2)$, the proposed class estimators reduces to the conventional unbiased estimator $\bar{y}^{*}$; that is, we have $P_{d(1 / 2,1 / 2)}=\bar{y}^{*}$. 


\section{ESTIMATION OF MEAN WITH TWO-PARAMETER...}

The recommended family $P_{d(\eta, \delta)}$ reduces to $T_{\mathrm{R} 2 d}=\bar{y}^{*}\left(\bar{x} / \bar{x}^{\prime}\right)$ for $(\eta, \delta)=(0,0)$ or $(1,1)$, and to $T_{\mathrm{R} 2 d}=\bar{y}^{*}\left(\bar{x} / \bar{x}^{\prime}\right)$ for $(\eta, \delta)=(1,0)$ or $(0,1)$. Write $e_{2}=(\bar{x}-\bar{X}) / \bar{X}$ such that $\mathrm{E}\left(e_{2}\right)=0, \mathrm{E}\left(e_{2}^{2}\right)=\lambda C_{x}^{2}, \mathrm{E}\left(e_{0} e_{2}\right)=\lambda \rho_{y x} C_{y} C_{x}$, and $\mathrm{E}\left(e_{2} e_{1}^{\prime}\right)=\lambda^{\prime} C_{x}^{2}$. Expressing (56),

$$
P_{d(\eta, \delta)}=\bar{Y}\left(1+e_{0}\right)\left[\eta \frac{1+(1-\delta) e_{2}+\delta e_{1}^{\prime}}{1+\delta e_{2}+(1-\delta) e_{1}^{\prime}}+(1-\eta) \frac{1+\delta e_{2}+(1-\delta) e_{1}^{\prime}}{1+(1-\delta) e_{2}+\delta e^{\prime}}\right]
$$

The expression (57) can be approximated as

$$
\begin{aligned}
P_{d(\eta, \delta)} \cong \bar{Y}[1+ & e_{0}-(1-2 \eta)(1-2 \delta)\left(e_{2}-e_{1}^{\prime}+e_{0} e_{2}-e_{0} e_{1}^{\prime}\right) \\
& \left.+(1-\eta-\delta)(1-2 \delta) e_{2}^{2}-(1-2 \delta)^{2} e_{2} e_{1}^{\prime}+(\eta-\delta) e_{1}^{\prime 2}\right]
\end{aligned}
$$

or

$$
\begin{aligned}
\left(P_{d(\eta, \delta)}-\bar{Y}\right) \cong \bar{Y}\left[e_{0}-\right. & (1-2 \eta)(1-2 \delta)\left(e_{2}-e_{1}^{\prime}+e_{0} e_{2}-e_{0} e_{1}^{\prime}\right) \\
& \left.+(1-\eta-\delta)(1-2 \delta) e_{2}^{2}-(1-2 \delta)^{2} e_{2} e_{1}^{\prime}+(\eta-\delta) e_{1}^{\prime 2}\right]
\end{aligned}
$$

The approximate bias of $P_{d(\eta, \delta)}$ is

$$
\begin{aligned}
\mathrm{B}\left(P_{d(\eta, \delta)}\right) & =\mathrm{E}\left(P_{d(\eta, \delta)}-\bar{Y}\right) \\
& =\bar{Y} C_{x}^{2}(1-2 \delta) \theta[(1-\eta-\delta)-(1-2 \eta) C]
\end{aligned}
$$

The suggested class of estimators $P_{d(\eta, \delta)}$ would be almost unbiased if

$$
\begin{aligned}
\mathrm{B}\left(P_{d(\eta, \delta)}\right)=0 & \Rightarrow(1-2 \delta)[(1-\eta-\delta)-(1-2 \eta) C]=0 \\
& \Rightarrow \delta=1 / 2 \text { or } \delta=1-\eta-C+2 \eta C
\end{aligned}
$$

If $\delta=1 / 2$ in (56), $P_{d(\eta, 1 / 2)}=\bar{y}^{*}$ (the conventional unbiased estimator), and for $\delta=1-\eta-C+2 \eta C$ in (56), $P_{d(\eta, \delta)}$ yields an almost unbiased estimator for $\bar{Y}$ as 


\section{PAL \& SINGH}

$$
\begin{aligned}
& P_{d(\eta)}=\bar{y}^{*}\left[\eta \frac{(\eta+C-2 \eta C) \bar{x}+(1-\eta-C+2 \eta C) \bar{X}}{(1-\eta-C+2 \eta C) \bar{x}+(\eta+C-2 \eta C) \bar{X}}\right. \\
& \left.+(1-\eta) \frac{(1-\eta-C+2 \eta C) \bar{x}+(\eta+C-2 \eta C) \bar{X}}{(\eta+C-2 \eta C) \bar{x}+(1-\eta-C+2 \eta C) \bar{X}}\right]
\end{aligned}
$$

The estimator (62) depends on the parameter $C$, which can be determined through a pilot sample survey. The bias of $P_{d(\eta, \delta)}$ is ignorable if the sample sizes $\left(n, n^{\prime}\right)$ approach the universe size $N$ because the factors $\lambda$ and $\lambda^{\prime}$ tend to zero. Squaring both sides of (57), the approximated expressions is

$$
\begin{aligned}
\left(P_{d(\eta, \delta)}-\bar{Y}\right)^{2} & \cong \bar{Y}^{2}\left[e_{0}-(1-2 \eta)(1-2 \delta)\left(e_{1}-e_{1}^{\prime}\right)\right]^{2} \\
= & \bar{Y}^{2}\left[e_{0}^{2}(1-2 \eta)^{2}(1-2 \delta)^{2}\left(e_{2}^{2}-2 e_{2} e_{1}^{\prime}+e_{1}^{\prime 2}\right)\right. \\
& \left.-2(1-2 \eta)(1-2 \delta)\left(e_{0} e_{2}-e_{0} e_{1}^{\prime}\right)\right]
\end{aligned}
$$

The approximate MSE of $P_{d(\eta, \delta)}$ is

$$
\begin{aligned}
\operatorname{MSE}\left(P_{d(\eta, \delta)}\right)=\bar{Y}^{2}\left[\lambda C_{y}^{2}\right. & +\lambda^{*} C_{y(2)}^{2} \\
& \left.+\theta(1-2 \eta)(1-2 \delta) C_{x}^{2}\{(1-2 \eta)(1-2 \delta)-2 C\}\right]
\end{aligned}
$$

which is minimum when

$$
(1-2 \eta)(1-2 \delta)=C
$$

The MMSE of $P_{d(\eta, \delta)}$ is given by

$$
\operatorname{MMSE}\left(P_{d(\eta, \delta)}\right)=\left[\lambda S_{y}^{2}\left(1-\rho_{y x}^{2}\right)+\lambda^{\prime} \rho_{y x}^{2} S_{y}^{2}+\lambda^{*} S_{y(2)}^{2}\right]
$$

Theorem 2. Up to $\mathrm{O}\left(n^{-1}\right)$,

$$
\operatorname{MSE}\left(P_{d(\eta, \delta)}\right) \geq\left[\lambda S_{y}^{2}\left(1-\rho_{y x}^{2}\right)+\lambda^{\prime} \rho_{y x}^{2} S_{y}^{2}+\lambda^{*} S_{y(2)}^{2}\right]
$$

if $(1-2 \eta)(1-2 \delta)=C$. 


\section{ESTIMATION OF MEAN WITH TWO-PARAMETER...}

Singh and Kumar (2009b) showed the quantity $\lambda S_{y}^{2}\left(1-\rho_{y x}^{2}\right)+\lambda^{\prime} \rho_{y x}^{2} S_{y}^{2}+\lambda^{*} S_{y(2)}^{2}$ is the minimal possible MSE, up to order $n^{-1}$, for a wide family of estimators to which the estimator (56) also belongs. For instance, for estimators of the form $P_{d \mathrm{~h}}=\bar{y}^{*} \mathrm{~h}(v)$ where $\mathrm{h}($.$) is a function of v$ such that $\mathrm{h}()=$.1 . Singh and Kumar (2009a) showed incorporating the sample and universe variances of $x$ might yield an estimator that has a lower MSE than $\lambda S_{y}^{2}\left(1-\rho_{y x}^{2}\right)+\lambda^{\prime} \rho_{y x}^{2} S_{y}^{2}+\lambda^{*} S_{y(2)}^{2}$, especially when relationship between $y$ and $x$ is markedly nonlinear. For every value of $C$, it is possible to select an AOE $P_{d(\eta, \delta)}^{(0)}$ from the two-parameter family in (56) (Chami et al., 2012, p. 6).

Remark 2. An alternative to the two-parameter RPR estimator defined in (56) is given by

$$
P_{d(\eta, \delta)}^{(1)}=\left[\eta v^{(1-2 \delta)}+(1-\eta) v^{(2 \delta-1)}\right] \bar{y}^{*}
$$

Up to order $n^{-1}$, the bias and MSE of $P_{d(\eta, \delta)}^{(1)}$ are same as defined for the family of $P_{d(\eta, \delta)}$. The class of estimators $P_{d(\eta, \delta)}^{(1)}$ is further generalized as

$$
P_{d(\eta, \delta)}^{(2)}=\left[\eta v^{(1-2 \delta)}+(1-\eta) v^{(2 \delta-1)}\right] \bar{y}^{*}
$$

where $v^{\prime}=(a \bar{x}+b) /\left(a \bar{x}^{\prime}+b\right)$ and $\eta, \delta, a$, and $b$ are the same as defined earlier.

\section{Efficiency Comparison and Choice of Parameter}

From (3) and (64),

$$
\begin{aligned}
\mathrm{V}\left(\bar{y}^{*}\right)-\operatorname{MSE}( & \left.P_{d(\eta, \delta)}\right) \\
& =\left(\lambda-\lambda^{\prime}\right) \bar{Y}^{2} C_{x}^{2}(1-2 \eta)(1-2 \delta)\{2 C-(1-2 \alpha)(1-2 \beta)\}
\end{aligned}
$$

which is positive if

$$
(1-2 \eta)(1-2 \delta)\{2 C-(1-2 \alpha)(1-2 \beta)\}>0
$$

Therefore, either 


\section{PAL \& SINGH}

(i) $\quad \eta>\frac{1}{2}, \delta>\frac{1}{2}$ and $\mathrm{C}>\frac{1}{2}(1-2 \eta)(1-2 \delta)$,

(ii) $\eta<\frac{1}{2}, \delta<\frac{1}{2}$ and $\mathrm{C}>\frac{1}{2}(1-2 \eta)(1-2 \delta)$,

(iii) $\eta<\frac{1}{2}, \delta>\frac{1}{2}$ and $\mathrm{C}<\frac{1}{2}(1-2 \eta)(1-2 \delta)$, or

(iv) $\eta>\frac{1}{2}, \delta>\frac{1}{2}$ and $\mathrm{C}<\frac{1}{2}(1-2 \eta)(1-2 \delta)$.

The family $P_{d(\eta, \delta)}$ is better than $\bar{y}^{*}$ as long as the above conditions hold true. It can be easily shown that $P_{d(\eta, \delta)}$ is more precise than

(i) $\quad T_{\mathrm{R} 2 d}$ (in the presence of non-response) if

$$
\text { either } C-1>(2 \eta \delta-\eta-\delta)>0
$$

or $\quad C-1<(2 \eta \delta-\eta-\delta)<0$

(ii) $T_{\mathrm{P} 2 d}$ (in the presence of non-response) if

either $C>(2 \eta \delta-\eta-\delta)>-1$

or $\quad C<(2 \eta \delta-\eta-\delta)<-1$

Remark 3. For a more explicit range of $\eta, \delta$, and $C$, the reader is referred to Chami et al. (2012).

\section{Unbiased Asymptotically Optimum Estimator}

Combining (61) and (64), calculate the parameters $\eta$ and $\delta$, where the suggested estimator becomes, at least up least up to $\mathrm{O}\left(n^{-1}\right)$, an unbiased AOE. Obtain a line with

$$
\delta=1 / 2, C=0
$$

(recall that on this line our estimator always reduces to $\bar{y}^{*}$ ) or a curve $\left(\eta^{*}(C), \delta^{*}(C), C\right) \in \mathrm{R}^{3}$ in the parameter space with

$$
\eta^{*}(C)=\frac{1}{2}(1 \pm \sqrt{C /(2 C-1)}), \delta^{*}(C)=\frac{1}{2}(1 \pm \sqrt{C(2 C-1)})
$$




\section{ESTIMATION OF MEAN WITH TWO-PARAMETER...}

The parametric curve in (69) is only defined for $C \leq 0$ or $C>1 / 2$. It is three hyperbolas. In the region $0<C \leq 1 / 2$ of the parameter space, minimizing MSE comes with a tradeoff in bias. Putting (70) in (56), the unbiased estimator for $\bar{Y}$ is

$$
P_{d\left(\eta^{*}(C), \delta^{*}(C)\right)}=\left[\frac{2(C+1) \bar{x}^{\prime}-2(C-1) \bar{x}^{2}+\left(2 C^{2}-C-1\right)\left(\bar{x}^{\prime}-\bar{x}\right)}{4 \bar{x}^{\prime} \bar{x}-\left(2 C^{2}-C-1\right)\left(\bar{x}^{\prime}-\bar{x}\right)^{2}}\right] \bar{y}^{*}
$$

The denominator vanishes if

$$
C=0.25\left(1 \pm \sqrt{9+\left(32 \bar{x}^{\prime} \bar{x}\right) /\left(\bar{x}^{\prime}-\bar{x}\right)^{2}}\right)
$$

It can be shown up to order $n^{-1}$ that the bias and MSE of the suggested estimators is

$$
\mathrm{B}\left(P_{d\left(\eta^{*}(C), \delta^{*}(C)\right)}\right)=0, \operatorname{MSE}\left(P_{d\left(\eta^{*}(C), \delta^{*}(C)\right)}\right)=\left[\lambda S_{y}^{2}\left(1-\rho_{y x}^{2}\right)+\lambda^{*} S_{y(2)}^{2}\right]
$$

Thus, the estimator $P_{d\left(\eta^{*}(C), \delta^{*}(C)\right)}$ is a biased AOE.

\section{Price Aspects on Both Cases}

\section{Derivation of Optimum Values of $n^{\prime}, n$, and $\boldsymbol{k}$ for Fixed Price $C^{\prime} \leq C_{0}$}

Denote the total (fixed) price of the surveys, apart from overhead, by $C_{0}$. The expected total price of the survey apart from overhead is given by

$$
C^{\prime}=c_{1}^{\prime} n^{\prime}+n\left(c_{1}+c_{2} W_{1}+\frac{c_{3} W_{2}}{k}\right)
$$

where $c_{1}^{\prime}$ is the price per unit of identifying and observing the supplementary character, $c_{1}$ is the price per unit of mailing a questionnaire/visiting the unit in the second-phase, $c_{2}$ is the price per unit of collecting or processing data obtained from the $n_{1}$ responding units, and $c_{3}$ is the price per unit of obtaining data for the subsampled units. For the sake of convenience of determination of $n^{\prime}, n$, and $k$ for (i) 


\section{PAL \& SINGH}

fixed price and (ii) specified MSE, retaining the terms of order $n^{-1}$, we write the approximate MSEs of the estimators $Q_{1}=T_{d(\alpha, \beta)}$ and $Q_{2}=P_{d(\eta, \delta)}$ as

$$
\operatorname{MSE}\left(Q_{i}\right)=\frac{1}{n} \bar{Y}^{2} V_{0 i}+\frac{1}{n^{\prime}} \bar{Y}^{2} V_{1 i}+\frac{k}{n} \bar{Y}^{2} V_{2 i}-\frac{1}{N} \bar{Y}^{2} C_{y}^{2}, \quad i=1,2,
$$

where $\bar{Y}^{2} V_{0 i}, \vec{Y}^{2} V_{1 i}$, and $\bar{Y}^{2} V_{2 i}$ are coefficients of the terms $1 / n, 1 / n^{\prime}$, and $k / n$ in $\operatorname{MSE}\left(Q_{i}\right), i=1,2$.

Consider a function $\varphi$ :

$$
\varphi=\operatorname{MSE}\left(Q_{i}\right)+\lambda_{i}\left\{c_{1}^{\prime} n^{\prime}+n\left(c_{1}+c_{2} W_{1}+\frac{c_{3} W_{2}}{k}\right)\right\}
$$

Using the calculus of obtaining the optimality,

$$
\begin{gathered}
n^{\prime}=\bar{Y} \sqrt{\frac{V_{1 i}}{\lambda_{i} c_{1}^{\prime}}}=\frac{1}{\lambda_{i}^{*}} \sqrt{\frac{V_{1 i}}{c_{1}^{\prime}}} \\
n=\bar{Y} \sqrt{\frac{V_{0 i}+k V_{1 i}}{\lambda_{i}\left(c_{1}+c_{2} W_{1}+\frac{c_{3} W_{2}}{k}\right)}}=\frac{1}{\lambda_{i}^{*}} \sqrt{\frac{V_{0 i}+k V_{1 i}}{c_{1}+c_{2} W_{1}+\frac{c_{3} W_{2}}{k}}} \\
\frac{n}{k}=\bar{Y} \sqrt{\frac{V_{2 i}}{\lambda_{i} c_{3} W_{2}}}=\frac{1}{\lambda_{i}^{*}} \sqrt{\frac{V_{2 i}}{c_{3} W_{2}}} .
\end{gathered}
$$

where

$$
\lambda_{i}^{*}=\frac{1}{C_{0}}\left[\sqrt{V_{1 i} c_{1}^{\prime}}+\sqrt{\left(V_{0 i}+k_{\mathrm{opt}}^{(i)} V_{2 i}\right)\left(c_{1}+c_{2} W_{1}+\frac{c_{3} W_{2}}{k_{\mathrm{opt}}^{(i)}}\right)}\right]
$$

The optimum value of $k$ is

$$
k_{\mathrm{opt}}^{(i)}=\sqrt{\frac{V_{0 i} c_{3} W_{2}}{\left(c_{1}+c_{2} W_{1}\right) V_{2 i}}} .
$$




\section{ESTIMATION OF MEAN WITH TWO-PARAMETER...}

With the help of (72), (75), (76), and (78),

$$
\bar{Y} \lambda_{i}^{*}=\sqrt{\lambda_{i}}
$$

The resulting minimum $\operatorname{MSE}\left(Q_{i}\right), i=1,2$, are

$$
\begin{aligned}
\operatorname{MMSE} & \left(Q_{i}\right) \\
& =\bar{Y}^{2}\left[\frac{1}{C_{0}}\left\{\sqrt{V_{1 i} c_{1}^{\prime}}+\sqrt{\left(V_{0 i}+k_{\mathrm{opt}}^{(i)} V_{2 i}\right)\left(c_{1}+c_{2} W_{1}+\frac{c_{3} W_{2}}{k_{\mathrm{opt}}^{(i)}}\right)}\right\}-\frac{1}{N} C_{y}^{2}\right]
\end{aligned}
$$

\section{Derivation of $n^{\prime}, n$, and $k$ for Specified MSE, $V \leq V_{0}^{*}$}

Observing (78), the optimum value of $k$ is independent of the total price or specified precision. Let $V_{0}^{*}$ be the fixed MSE of $Q_{i}, i=1,2$ :

$$
V_{0}^{*}=\bar{Y}^{2}\left[\frac{1}{n} V_{0 i}+\frac{1}{n^{\prime}} V_{1 i}+\frac{k}{n} V_{2 i}-\frac{1}{N} C_{y}^{2}\right], \quad i=1,2
$$

Thus,

$$
\begin{aligned}
& \sqrt{\frac{1}{\lambda_{i}}} \\
& =\bar{Y}^{2}\left[\frac{1}{C_{0}}\left\{\sqrt{V_{1 i} c_{1}^{\prime}}+\sqrt{\left(V_{0 i}+k_{\mathrm{opt}}^{(i)} V_{2 i}\right)\left(c_{1}+c_{2} W_{1}+\frac{c_{3} W_{2}}{k_{\mathrm{opt}}^{(i)}}\right)}\right\}\right]\left[V_{0}^{*}+\frac{\bar{Y}^{2} C_{y}^{2}}{N}\right]^{-1}
\end{aligned}
$$

Using the optimum value of the expected price, obtain the MSE

$$
\begin{aligned}
& \mathrm{D}\left(Q_{i}\right) \\
& =\bar{Y}^{2}\left[\frac{1}{C_{0}}\left\{\sqrt{V_{1 i} c_{1}^{\prime}}+\sqrt{\left(V_{0 i}+k_{\mathrm{opt}}^{(i)} V_{2 i}\right)\left(c_{1}+c_{2} W_{1}+\frac{c_{3} W_{2}}{k_{\mathrm{opt}}^{(i)}}\right)}\right\}\right]^{2}\left[V_{0}^{*}+\frac{\bar{Y}^{2} C_{y}^{2}}{N}\right]^{-1}
\end{aligned}
$$




\section{PAL \& SINGH}

\section{Numerical Example}

Population source: Khare and Sinha (2007). The values of required parameters are

$$
\begin{aligned}
& N=95, n=35, n^{\prime}=70, D_{1}=0.75, D_{2}=0.25, C_{y}=0.15613, C_{(y)}=0.12075, \\
& C_{x}=0.03006, C_{x(2)}=0.02478, \rho_{y x}=0.328, C_{y x(2)}=0.477
\end{aligned}
$$

\section{Case I: There Is Non-Response on $y$ as Well as on $\boldsymbol{x}$}

Compute the optimum values of $\alpha$ for given $k$ and $\beta$ by using the formula

$$
\alpha_{\mathrm{opt}}=1 / 2\left[1-\left\{R_{d}^{*} /(1-2 \beta)\right\}\right]
$$

for $k=5(-1) 2$ and $\beta(>1 / 2)=0.51,0.75,1.00(0.25) 2.50, \beta(<1 / 2)=0.49,0.25$, $0.00(0.25)-1.50$. Findings are shown in Table 1 . However, the optimum values of $\beta$ for given $k$ and $\alpha$ may also be computed by using the formula

$$
\beta_{\mathrm{opt}}=1 / 2\left[1-\left\{R_{d}^{*} /(1-2 \alpha)\right\}\right] .
$$

Table 1. Optimum values of $\alpha$ for selected values of $\beta$ and for $k=5(-1) 2$

\begin{tabular}{rrrrrrrrrr} 
& \multicolumn{10}{c}{$\boldsymbol{\beta}$} \\
\cline { 2 - 10 } & $\mathbf{0 . 5 1}$ & $\mathbf{0 . 7 5}$ & $\mathbf{1 . 0 0}$ & $\mathbf{1 . 2 5}$ & $\mathbf{1 . 5 0}$ & $\mathbf{1 . 7 5}$ & $\mathbf{2 . 0 0}$ & $\mathbf{2 . 2 5}$ & $\mathbf{2 . 5 0}$ \\
\hline 5 & 52.0309 & 2.5612 & 1.5306 & 1.1871 & 1.0153 & 0.9122 & 0.8435 & 0.7945 & 0.7577 \\
4 & 50.9240 & 2.5170 & 1.5085 & 1.1723 & 1.0042 & 0.9034 & 0.8362 & 0.7881 & 0.7521 \\
3 & 49.3693 & 2.4548 & 1.4774 & 1.1516 & 0.9887 & 0.8910 & 0.8258 & 0.7793 & 0.7443 \\
2 & 47.0260 & 2.3610 & 1.4305 & 1.1203 & 0.9653 & 0.8722 & 0.8102 & 0.7659 & 0.7326 \\
\hline
\end{tabular}

\begin{tabular}{rrrrrrrrrr}
$\boldsymbol{k}$ & $\mathbf{0 . 4 9}$ & $\mathbf{0 . 2 5}$ & $\mathbf{0 . 0 0}$ & $\mathbf{- 0 . 2 5}$ & $\mathbf{- 0 . 5 0}$ & $\mathbf{- 0 . 7 5}$ & $\mathbf{- 1 . 0 0}$ & $\mathbf{- 1 . 2 5}$ & $\mathbf{- 1 . 5 0}$ \\
\hline 5 & -51.0309 & -1.5612 & -0.5306 & -0.1871 & -0.0153 & 0.0878 & 0.1565 & 0.2055 & 0.2423 \\
4 & -49.9240 & -1.5170 & -0.5085 & -0.1723 & -0.0042 & 0.0966 & 0.1638 & 0.2119 & 0.2479 \\
3 & -48.3693 & -1.4548 & -0.4774 & -0.1516 & 0.0113 & 0.1090 & 0.1742 & 0.2207 & 0.2557 \\
2 & -46.0260 & -1.3610 & -0.4305 & -0.1203 & 0.0347 & 0.1278 & 0.1898 & 0.2341 & 0.2674 \\
\hline
\end{tabular}


ESTIMATION OF MEAN WITH TWO-PARAMETER...

Table 2. The PRE of $T_{d(\alpha, \beta)}$ with respect to $\vec{y}$

\begin{tabular}{|c|c|c|c|c|c|c|c|c|c|c|}
\hline & \multirow[b]{2}{*}{$\beta$} & \\
\hline$k$ & & 0.51 or 0.49 & 0.75 or 0.25 & 1.00 or 0.00 & 1.25 or -0.25 & 1.50 or -0.50 & 1.75 or -0.75 & 2.00 or -1.00 & 2.25 or -1.25 & 2.50 or -1.50 \\
\hline \multirow[t]{9}{*}{5} & 0.51 or 0.49 & 100.0059 & 100.1464 & 100.2926 & 100.4385 & 100.5840 & 100.7293 & 100.8743 & 101.0189 & 101.1633 \\
\hline & 0.75 or 0.25 & 100.1464 & 103.5650 & 106.8834 & 109.8822 & 112.4890 & 114.6353 & 116.2613 & 117.3191 & 117.7766 \\
\hline & 1.00 or 0.00 & 100.2926 & 106.8834 & 112.4890 & 116.2613 & 117.7766 & 116.8529 & 113.6019 & 108.3970 & 101.7727 \\
\hline & 1.25 or -0.25 & 100.4385 & 109.8822 & 116.2613 & 117.6196 & 113.6019 & 105.2259 & * & * & * \\
\hline & 1.50 or -0.50 & 100.5840 & 112.4890 & 117.7766 & 113.6019 & 101.7727 & * & * & * & * \\
\hline & 1.75 or -0.75 & 100.7293 & 114.6353 & 116.8529 & 105.2259 & * & * & * & * & * \\
\hline & 2.00 or -1.00 & 100.8743 & 116.2613 & 113.6019 & * & * & * & * & * & * \\
\hline & 2.25 or -1.25 & 101.0189 & 117.3191 & 108.3970 & * & * & * & * & * & * \\
\hline & 2.50 or -1.50 & 101.1633 & 117.7766 & 101.7727 & * & * & * & * & * & * \\
\hline \multirow[t]{9}{*}{4} & 0.51 or 0.49 & 100.0056 & 100.1396 & 100.2789 & 100.4179 & 100.5566 & 100.6950 & 100.8330 & 100.9707 & 101.1081 \\
\hline & 0.75 or 0.25 & 100.1396 & 103.3888 & 106.5215 & 109.3299 & 111.7470 & 113.7105 & 115.1669 & 116.0747 & 116.4070 \\
\hline & 1.00 or 0.00 & 100.2789 & 106.5215 & 111.7470 & 115.1669 & 116.4070 & 115.3230 & 112.0413 & 106.9231 & 100.4724 \\
\hline & 1.25 or -0.25 & 100.4179 & 109.3299 & 115.1669 & 116.1539 & 112.0413 & 103.8304 & * & * & * \\
\hline & 1.50 or -0.50 & 100.5566 & 111.7470 & 116.4070 & 112.0413 & 100.4724 & * & * & * & * \\
\hline & 1.75 or -0.75 & 100.6950 & 113.7105 & 115.3230 & 103.8304 & * & * & * & * & * \\
\hline & 2.00 or -1.00 & 100.8330 & 115.1669 & 112.0413 & * & * & * & * & * & * \\
\hline & 2.25 or -1.25 & 100.9707 & 116.0747 & 106.9231 & * & * & * & * & * & * \\
\hline & 2.50 or -1.50 & 101.1081 & 116.4070 & 100.4724 & * & * & * & * & * & * \\
\hline
\end{tabular}

Note: * indicates the PRE was less than 100 
PAL \& SINGH

Table 2 (continued).

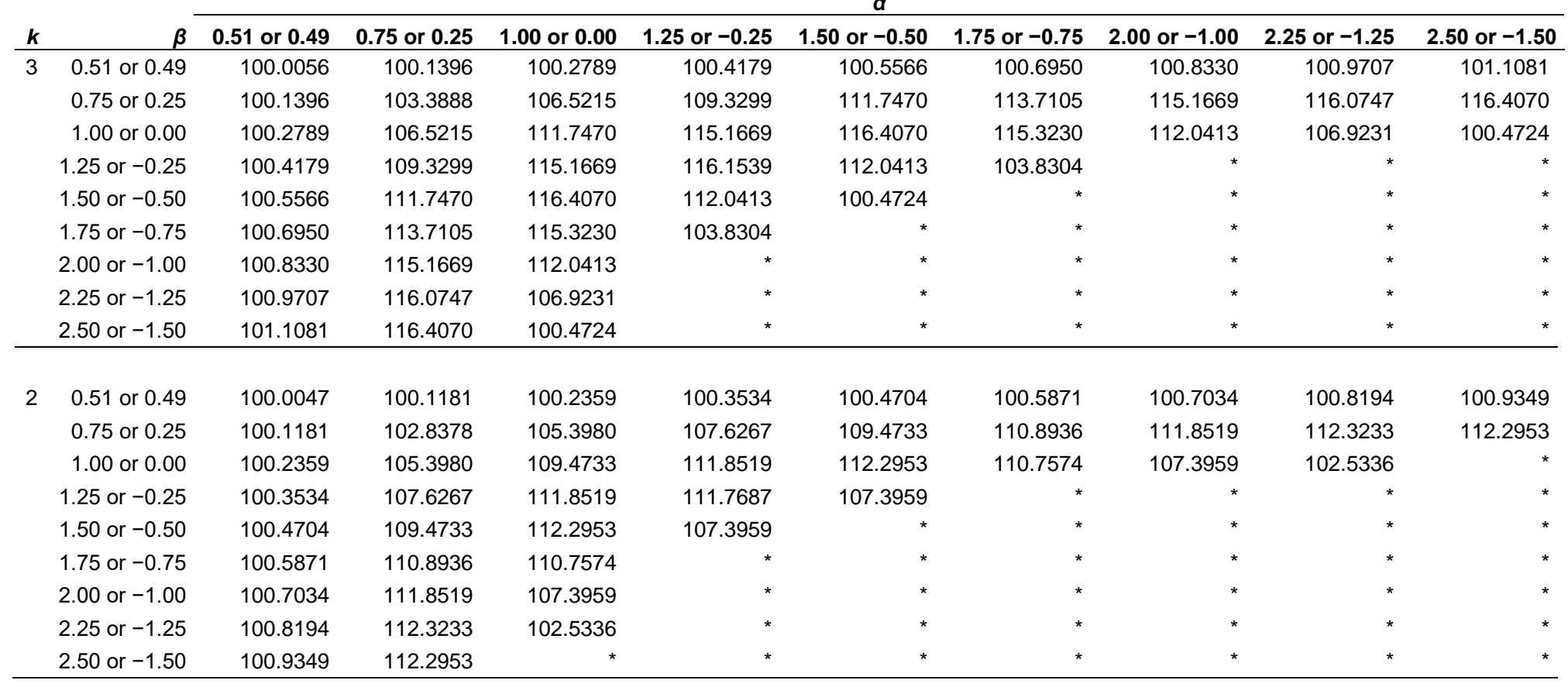

Note: * indicates the PRE was less than 100 


\section{ESTIMATION OF MEAN WITH TWO-PARAMETER...}

Table 3. PREs of AOE $T_{d(\alpha, \beta)}^{(0)}$ and of $T_{\mathrm{R} 1 d}$ with respect to $\bar{y}^{*}$

\begin{tabular}{rrrrrr}
$\boldsymbol{k}$ & $\mathbf{5}$ & $\mathbf{4}$ & $\mathbf{3}$ & $\mathbf{2}$ \\
\hline $\operatorname{PRE}\left(T_{d(\alpha, \beta)}^{(0)}, \bar{y}^{*}\right)$ & 117.7951 & 116.4084 & 114.6544 & 112.3728 \\
$\operatorname{PRE}\left(T_{\mathrm{R} 1 d,} \bar{y}^{*}\right)$ & 112.4890 & 111.7470 & 110.7814 & 109.4733 \\
\hline
\end{tabular}

The percent relative efficiency (PRE) of $T_{d(\alpha, \beta)}$ with respect to $\bar{y}^{*}$ is obtained using the formula, the results of which are given in Table 2.

$$
\begin{aligned}
& \operatorname{PRE}\left(T_{d(\alpha, \beta)}, \bar{y}^{*}\right) \\
& =\left(\lambda C_{y}^{2}+\lambda^{*} C_{y(2)}^{2}\right)\left(\lambda C_{y}^{2}+\lambda^{*} C_{y(2)}^{2}\right. \\
& \left.\quad+(1-2 \alpha)(1-2 \beta)\left(\theta C_{x}^{2}+\lambda^{*} C_{x(2)}^{2}\right)\left[(1-2 \alpha)(1-2 \beta)-2 R_{d}^{*}\right]\right)^{-1} \times 100
\end{aligned}
$$

The PREs of the AOE $T_{d(\alpha, \beta)}^{(0)}$ and of $T_{\mathrm{R} 1 d}$ with respect to $\bar{y}^{*}$ are obtained using the formulae

$$
\begin{gathered}
\operatorname{PRE}\left(T_{d(\alpha, \beta)}^{(0)}, \bar{y}^{*}\right)=\frac{\lambda C_{y}^{2}+\lambda^{*} C_{y(2)}^{2}}{\left(\lambda C_{y}^{2}+\lambda^{*} C_{y(2)}^{2}\right)-\left(\theta C_{x}^{2}+\lambda^{*} C_{x(2)}^{2}\right) R_{d}^{* 2}} \times 100 \\
\operatorname{PRE}\left(T_{\mathrm{R} 1 d}, \bar{y}^{*}\right)=\frac{\lambda C_{y}^{2}+\lambda^{*} C_{y(2)}^{2}}{\left(\lambda C_{y}^{2}+\lambda^{*} C_{y(2)}^{2}\right)-\left(\theta C_{x}^{2}+\lambda^{*} C_{x(2)}^{2}\right)\left(1-2 R_{d}^{* 2}\right)} \times 100
\end{gathered}
$$

Findings are given Table 3.

Observe from Table 1 that (i) when $k$ is fixed, $\alpha_{\text {opt }}$ decreases when $\beta(>1 / 2)$ increases up to 2.50; (ii) for fixed values of $k$, the magnitude of $\alpha_{\text {opt }}$ (i.e., absolute optimum value of $\alpha$ ) decreases when $\beta(<1 / 2)$ decreases to -1.50 . Table 2 shows that the PRE of $T_{d(\alpha, \beta)}$ with respect to $\bar{y}^{*}$ is larger than 100 for $(\alpha, \beta) \in(0.51,2.50)$, $(\alpha, \beta) \in(-1.50,0.49)$, and all values of $k=5(-1) 2$. Thus it follows that, in said range of $(\alpha, \beta)$ and all the values of $k=5(-1) 2$, the suggested estimator $T_{d(\alpha, \beta)}$ is more accurate than $\bar{y}^{*}$. A large number of flexible values of $(\alpha, \beta)$ exist for which the suggested estimator is superior to $\bar{y}^{*}$. From Table 3 , observe the AOE $T_{d(\alpha, \beta)}^{(0)}$ is more accurate than $\bar{y}^{*}$ and $T_{\mathrm{R} 1 d}$ with a substantial gain in efficiency. 


\section{PAL \& SINGH}

Table 4. Optimum values of $\eta$ for given $\delta$

\begin{tabular}{rrrrrrrrrr}
$\boldsymbol{\delta}$ & $\mathbf{0 . 5 1}$ & $\mathbf{0 . 7 5}$ & $\mathbf{1 . 0 0}$ & $\mathbf{1 . 2 5}$ & $\mathbf{1 . 5 0}$ & $\mathbf{1 . 7 5}$ & $\mathbf{2 . 0 0}$ & $\mathbf{2 . 2 5}$ & $\mathbf{2 . 5 0}$ \\
\hline$\eta_{\text {opt }}$ & 43.09040 & 2.20360 & 1.35180 & 1.06790 & 0.92591 & 0.84070 & 0.78390 & 0.74340 & 0.71300 \\
\hline
\end{tabular}

\begin{tabular}{rrrrrrrrrr}
$\delta$ & $\mathbf{0 . 4 9}$ & $\mathbf{0 . 2 5}$ & $\mathbf{0 . 0 0}$ & $\mathbf{- 0 . 2 5}$ & $-\mathbf{0 . 5 0}$ & $\mathbf{- 0 . 7 5}$ & $\mathbf{- 1 . 0 0}$ & $\mathbf{- 1 . 2 5}$ & $\mathbf{- 1 . 5 0}$ \\
\hline$\eta_{\text {opt }}$ & -42.09040 & -1.20360 & -0.35180 & -0.06790 & 0.07410 & 0.15930 & 0.21610 & 0.25660 & 0.28700 \\
\hline
\end{tabular}

Case II: When NR Occurs Only on $y$ and Information on $x$ is Available

Compute the optimum values of $\eta$ for a given $\delta$ using the following formula:

$$
\eta_{\mathrm{opt}}=1 / 2[1-\{C /(1-2 \delta)\}]
$$

The results are given in Table 4.

The optimum values of $\delta$ for a given $\eta$ may also be computed by using the following formula:

$$
\delta_{\text {opt }}=1 / 2[1-\{C /(1-2 \eta)\}]
$$

The PRE of $P_{d(\eta, \delta)}$ with respect to $\bar{y}^{*}$ is obtained using the formula

$$
\begin{aligned}
& \operatorname{PRE}\left(P_{d(\eta, \delta)}, \bar{y}^{*}\right) \\
& =\left(\lambda C_{y}^{2}+\lambda^{*} C_{y(2)}^{2}\right)\left(\lambda C_{y}^{2}+\lambda^{*} C_{y(2)}^{2}\right. \\
& \left.\quad+(1-2 \eta)(1-2 \delta)\left(\theta C_{x}^{2}+\lambda^{*} C_{x(2)}^{2}\right)[(1-2 \eta)(1-2 \delta)-2 C]\right)^{-1} \times 100
\end{aligned}
$$

The results are given in Table 5. The PREs of the AOE $P_{d(\eta, \delta)}^{(0)}$ and of $T_{\mathrm{R} 2 d}$ with respect to $\bar{y}^{*}$ are obtained using the formulae below, the results are given Table 6 .

$$
\begin{gathered}
\operatorname{PRE}\left(P_{d(\eta, \delta)}^{(0)}, \bar{y}^{*}\right)=\frac{\lambda C_{y}^{2}+\lambda^{*} C_{y(2)}^{2}}{\left(\lambda C_{y}^{2}+\lambda^{*} C_{y(2)}^{2}\right)-\theta C^{2} C_{x}^{2}} \times 100 \\
\operatorname{PRE}\left(P_{\mathrm{R} 2 d}, \bar{y}^{*}\right)=\frac{\lambda C_{y}^{2}+\lambda^{*} C_{y(2)}^{2}}{\left(\lambda C_{y}^{2}+\lambda^{*} C_{y(2)}^{2}\right)-\theta C_{x}^{2}\left(1-2 C^{2}\right)} \times 100
\end{gathered}
$$


ESTIMATION OF MEAN WITH TWO-PARAMETER...

Table 5. The PRE of $P_{d(\eta, \delta)}$ with respect to $\vec{y}$

\begin{tabular}{|c|c|c|c|c|c|c|c|c|c|c|}
\hline \multirow[b]{2}{*}{$k$} & \multirow[b]{2}{*}{$\delta$} & \multicolumn{9}{|c|}{$\eta$} \\
\hline & & 0.51 or 0.49 & 0.75 or 0.25 & 1.00 or 0.00 & 1.25 or -0.25 & 1.50 or -0.50 & 1.75 or -0.75 & 2.00 or -1.00 & 2.25 or -1.25 & 2.50 or -1.50 \\
\hline \multirow[t]{9}{*}{5} & 0.51 or 0.49 & 100.0021 & 100.0512 & 100.1022 & 100.1529 & 100.2034 & 100.2536 & 100.3036 & 100.3533 & 100.4028 \\
\hline & 0.75 or 0.25 & 100.0512 & 101.2040 & 102.2400 & 103.0967 & 103.7648 & 104.2364 & 104.5062 & 104.5709 & 104.4299 \\
\hline & 1.00 or 0.00 & 100.1022 & 102.2400 & 103.7648 & 104.5062 & 104.4299 & 103.5394 & 101.8759 & * & * \\
\hline & 1.25 or -0.25 & 100.1529 & 103.0967 & 104.5062 & 104.0847 & 101.8759 & * & * & * & * \\
\hline & 1.50 or -0.50 & 100.2034 & 103.7648 & 104.4299 & 101.8759 & * & * & * & * & * \\
\hline & 1.75 or -0.75 & 100.2536 & 104.2364 & 103.5394 & * & * & * & * & * & * \\
\hline & 2.00 or -1.00 & 100.3036 & 104.5062 & 101.8759 & * & * & * & * & * & * \\
\hline & 2.25 or -1.25 & 100.3533 & 104.5709 & * & * & * & * & * & * & * \\
\hline & 2.50 or -1.50 & 100.4028 & 104.4299 & * & * & * & * & * & * & * \\
\hline \multirow[t]{9}{*}{4} & 0.51 or 0.49 & 100.0023 & 100.0583 & 100.1164 & 100.1741 & 100.2316 & 100.2889 & 100.3458 & 100.4024 & 100.4588 \\
\hline & 0.75 or 0.25 & 100.0583 & 101.3729 & 102.5580 & 103.5406 & 104.3084 & 104.8513 & 105.1622 & 105.2368 & 105.0742 \\
\hline & 1.00 or 0.00 & 100.1164 & 102.5580 & 104.3084 & 105.1622 & 105.0742 & 104.0492 & 102.1411 & * & * \\
\hline & 1.25 or -0.25 & 100.1741 & 103.5406 & 105.1622 & 104.6766 & 102.1411 & * & * & * & * \\
\hline & 1.50 or -0.50 & 100.2316 & 104.3084 & 105.0742 & 102.1411 & * & * & * & * & * \\
\hline & 1.75 or -0.75 & 100.2889 & 104.8513 & 104.0492 & * & * & * & * & * & * \\
\hline & 2.00 or -1.00 & 100.3458 & 105.1622 & 102.1411 & * & * & * & * & * & * \\
\hline & 2.25 or -1.25 & 100.4024 & 105.2368 & * & * & * & * & * & * & * \\
\hline & 2.50 or -1.50 & 100.4588 & 105.0742 & * & * & * & * & * & * & * \\
\hline
\end{tabular}

Note: * indicates the PRE was less than 100 
PAL \& SINGH

Table 5 (continued).

\begin{tabular}{|c|c|c|c|c|c|c|c|c|c|c|}
\hline \multirow[b]{2}{*}{$k$} & & \multicolumn{9}{|c|}{$\eta$} \\
\hline & $\delta$ & 0.51 or 0.49 & 0.75 or 0.25 & 1.00 or 0.00 & 1.25 or -0.25 & 1.50 or -0.50 & 1.75 or -0.75 & 2.00 or -1.00 & 2.25 or -1.25 & 2.50 or -1.50 \\
\hline \multirow[t]{9}{*}{3} & 0.51 or 0.49 & 100.0027 & 100.0677 & 100.1351 & 100.2022 & 100.2690 & 100.3354 & 100.4016 & 100.4674 & 100.5329 \\
\hline & 0.75 or 0.25 & 100.0677 & 101.5970 & 102.9812 & 104.1330 & 105.0355 & 105.6750 & 106.0417 & 106.1298 & 105.9379 \\
\hline & 1.00 or 0.00 & 100.1351 & 102.9812 & 105.0355 & 106.0417 & 105.9379 & 104.7306 & 102.4937 & * & * \\
\hline & 1.25 or -0.25 & 100.2022 & 104.1330 & 106.0417 & 105.4691 & 102.4937 & * & * & * & * \\
\hline & 1.50 or -0.50 & 100.2690 & 105.0355 & 105.9379 & 102.4937 & * & * & * & * & * \\
\hline & 1.75 or -0.75 & 100.3354 & 105.6750 & 104.7306 & * & * & * & * & * & * \\
\hline & 2.00 or -1.00 & 100.4016 & 106.0417 & 102.4937 & * & * & * & * & * & * \\
\hline & 2.25 or -1.25 & 100.4674 & 106.1298 & * & * & * & * & * & * & * \\
\hline & 2.50 or -1.50 & 100.5329 & 105.9379 & * & * & * & * & * & * & * \\
\hline \multirow[t]{9}{*}{2} & 0.51 or 0.49 & 100.0032 & 100.0807 & 100.1610 & 100.2410 & 100.3206 & 100.3999 & 100.4788 & 100.5574 & 100.6356 \\
\hline & 0.75 or 0.25 & 100.0807 & 101.9086 & 103.5724 & 104.9635 & 106.0579 & 106.8357 & 107.2826 & 107.3900 & 107.1560 \\
\hline & 1.00 or 0.00 & 100.1610 & 103.5724 & 106.0579 & 107.2826 & 107.1560 & 105.6878 & 102.9854 & * & * \\
\hline & 1.25 or -0.25 & 100.2410 & 104.9635 & 107.2826 & 106.5850 & 102.9854 & * & * & * & * \\
\hline & 1.50 or -0.50 & 100.3206 & 106.0579 & 107.1560 & 102.9854 & * & * & * & * & * \\
\hline & 1.75 or -0.75 & 100.3999 & 106.8357 & 105.6878 & * & * & * & * & * & * \\
\hline & 2.00 or -1.00 & 100.4788 & 107.2826 & 102.9854 & * & * & * & * & * & * \\
\hline & 2.25 or -1.25 & 100.5574 & 107.3900 & * & * & * & * & * & * & * \\
\hline & 2.50 or -1.50 & 100.6356 & 107.1560 & * & * & * & * & * & * & * \\
\hline
\end{tabular}

Note: * indicates the PRE was less than 100 


\section{ESTIMATION OF MEAN WITH TWO-PARAMETER...}

Table 6. PREs of AOE $P_{d(\eta, \bar{\delta})}^{(0)}$ and of $T_{\mathrm{R} 2 d}$ with respect to $\bar{y}^{*}$

\begin{tabular}{rrrrr}
$\boldsymbol{k}$ & $\mathbf{5}$ & $\mathbf{4}$ & $\mathbf{3}$ & $\mathbf{2}$ \\
\hline $\operatorname{PRE}\left(P_{d(\eta, \overline{)}}^{(0)}, \bar{y}^{*}\right)$ & 104.5744 & 105.2409 & 106.1346 & 107.3959 \\
$\operatorname{PRE}\left(T_{\mathrm{R} 2 d,}, \bar{y}^{*}\right)$ & 103.7648 & 104.3084 & 105.0355 & 106.0529 \\
\hline
\end{tabular}

Table 4 exhibits that (i) the optimum value of $\eta$ decreases as $\delta(>1 / 2)$ increases up to 2.50; (ii) the absolute of optimum value of $\eta$ also decreases when $\delta(<1 / 2)$ decreases to -1.50 . Observe from Table 5 that (i) for $-1.50 \leq \eta \leq 2.50$, $0.51 \leq \delta \leq 1.00$, and $k=5(-1) 2$, the proposed class of estimators $P_{d(\eta, \delta)}$ are always better than $\bar{y}^{*}$; (ii) for $-0.50 \leq \eta, \delta \leq 1.50$ and $k=5(-1) 2$, the envisaged estimator $P_{d(\eta, \delta)}$ is more efficient than $\bar{y}^{*}$ with a considerable gain in efficiency. Table 6 shows that the envisaged AOE $P_{d(\eta, \delta)}^{(0)}$ is more efficient than $\bar{y}^{*}$ and $T_{\mathrm{R} 2 d}$ for $k=5(-1) 2$.

From Table 2, note there is enough flexibility in choosing the values of the scalars $\eta, \delta$ in order to get estimators $\eta, \delta$ for $P_{d(\eta, \delta)}$ and better than $\bar{y}^{*}, T_{\mathrm{R} 2 d}$. It is also observed from Table 5 that, for fixed values of $\eta, \delta$, the values of $\operatorname{PRE}\left(P_{d(\eta, \delta)}, \bar{y}^{*}\right)$ increase as the values of $k$ decrease. Comparing the results shown in Tables 2 and 3 to Tables 5 and 6 , the proposed family of estimators $T_{d(\alpha, \beta)}$ (where NR occurs on both the variables $y, x)$ performs better than the corresponding estimator $P_{d(\eta, \delta)}$ (where NR occurs only on the main variable $y$ ). The recommendation favors that the suggested estimators $T_{d(\alpha, \beta)}$ and $P_{d(\eta, \delta)}$ be used in practice.

\section{Acknowledgements}

The authors would like to thank the Editor and the three anonymous referees for carefully reading the paper and their valuable suggestions.

\section{References}

Chami, S. P., Sing, B., \& Thomas, D. (2012). A two-parameter ratioproduct-ratio estimator using auxiliary information. ISRN Probability and Statistics, 2012, Article ID 103860. doi: 10.5402/2012/103860

Cochran, W. G. (1977). Sampling techniques (3rd ed.). New York, NY: John Wiley and Sons. 


\section{PAL \& SINGH}

Hansen, M. H., \& Hurwitz, W.N. (1946). The problem of non-response in sample surveys. Journal of the American Statistical Association, 41(236), $517-$ 529. doi: 10.1080/01621459.1946.10501894

Khare, B. B., \& Sinha, R. R. (2007). Estimation of the ratio of the two population using multi-auxiliary characters in the presence of non-response. In B. N. Pandey (Ed.), Statistical techniques in life-testing, reliability, sampling theory and quality control (pp.163-171). New Delhi, India: Narosa Publishing House.

Khare, B. B., \& Srivastava, S. (1993). Estimation of population mean using auxiliary character in presence of non-response. National Academy Science Letters, 16, 111-114.

Khare, B. B., \& Srivastava, S. (1995). Study of conventional and alternative two-phase sampling ratio, product and regression estimators in presence of nonresponse. Proceedings - National Academy of Sciences India, 65, 195-203.

Pal, S. K., \& Singh, H. P. (2016). Finite population mean estimation through a two-parameter ratio estimator using auxiliary information in presence of nonresponse. Journal of Applied Mathematics, Statistics and Informmatics, 12(2), 538. doi: 10.1515/jamsi-2016-0006

Pal, S. K., \& Singh, H. P. (2017). A class of ratio-cum-ratio-type exponential estimators for population mean with sub-sampling the nonrespondents. Jordan Journal of Mathematics and Statistics, 10(1), 73-94. Retrieved from http://journals.yu.edu.jo/jjms/Issues/Vol10No12017PDF/5.pdf

Rao, P. S. R. S. (1986). Ratio estimation with sub-sampling the nonrespondents. Survey Methodology, 12(2), 217-230.

Singh, H. P., \& Kumar, S. (2008). A regression approach to the estimation of the finite population mean in the presence of non-response. Australian \& New Zealand Journal of Statistics, 50(4), 395-408. doi: 10.1111/j.1467-

842x.2008.00525.x

Singh, H. P., \& Kumar, S. (2009a). A general class of estimators of the population mean in survey sampling using auxiliary information with sub sampling the non- respondents. Korean Journal of Applied Statistics, 22(2), 387402. doi: 10.5351/kjas.2009.22.2.387

Singh, H. P., \& Kumar, S. (2009b). A general procedure of estimating the population mean in the presence of non-response under double sampling using auxiliary information. SORT, 33(1), 71-84. Retrieved from https://www.raco.cat/index.php/SORT/article/view/144072 


\section{ESTIMATION OF MEAN WITH TWO-PARAMETER...}

Singh, H. P., \& Kumar, S. (2010a). Estimation of mean in presence of nonresponse using two phase sampling scheme. Statistical Papers, 51(3), 559-582. doi: 10.1007/s00362-008-0140-5

Singh, H. P., \& Kumar, S. (2010b). Improved estimation of population mean under two-phase sampling with subsampling the non-respondent. Journal of Statistical Planning and Inference, 140(9), 2536-2550. doi: 10.1016/j.jspi.2010.03.023

Singh, H. P., Kumar, S., \& Kozak, M. (2010). Improved estimation of finitepopulation mean using sub-sampling to deal with non response in two-phase sampling. Communications in Statistics - Theory and Methods, 39(5), 791-802. doi: 10.1080/03610920902796056

Singh, H. P., Solanki, R. S., \& Singh, A. K. (2016). A generalized ratiocum-product estimator for estimating the finite population mean in survey sampling. Communications in Statistics - Theory and Methods, 45(1), 158-172. doi: 10.1080/03610926.2013.827719

Srivastava, S. K. (1971). A generalized estimator using multi-auxiliary information. Journal of the American Statistical Association, 66(334), 404-407. doi: 10.1080/01621459.1971.10482277

Tabasum, R., \& Khan, I. A. (2004). Double sampling for ratio estimation with non-response. Journal of the Indian Society of Agricultural Statistics, 58(3), 300-306. Retrieved from http://isas.org.in/jsp/volume/vol58/issue3/rifattabasum.pdf

Wu, C., \& Luan, Y. (2003). Optimal calibration estimators under two-phase sampling. Journal of Official Statistics, 19(2), 119-131. 